\title{
SENSITIVITY ANALYSIS OF A PARABOLIC-ELLIPTIC PROBLEM
}

\author{
BY \\ BASTIAN GEBAUER
}

\begin{abstract}
Johann Radon Institute for Computational and Applied Mathematics (RICAM), Austrian Academy of Sciences, Altenbergerstr. 69, 4040 Linz, Austria
\end{abstract}

\begin{abstract}
We consider the heat flux through a domain with subregions in which the thermal capacity approaches zero. In these subregions the parabolic heat equation degenerates to an elliptic one. We show the well-posedness of such parabolic-elliptic differential equations for general non-negative $L^{\infty}$-capacities and study the continuity of the solutions with respect to the capacity, thus giving a rigorous justification for modeling a small thermal capacity by setting it to zero. We also characterize weak directional derivatives of the temperature with respect to capacity as solutions of related parabolic-elliptic problems.
\end{abstract}

1. Introduction. The temperature $u(x, t)$ inside a domain $B$ that is subject to an external heating force $f(x, t)$ is described by the well-known heat equation

$$
\left.\partial_{t}(c(x) u(x, t))-\nabla \cdot(\kappa(x) \nabla u(x, t))=f(x, t), \quad(x, t) \in B \times\right] 0, T[,
$$

where $\kappa(x)$ is the thermal conductivity and $c(x)$ the thermal capacity of the domain.

As long as the heat capacity $c$ is bounded from below by a positive constant the equation is of parabolic type. However, there are situations where the capacity seems negligibly small in some subset of $B$. As an example we might think of regions of high capacity in a body with otherwise low capacity, such as wet areas in an otherwise dry background. Due to their high capacity the watery parts slowly heat up or cool down, while the rest of the domain almost instantly reaches its state of thermal equilibrium.

If we formally set $c(x)=\chi_{\Omega}(x)$, where $\chi_{\Omega}$ is the characteristic function of some open subdomain $\Omega \subseteq B$ with smooth boundary $\partial \Omega$ and assume that $\kappa(x)=1$, then (1.1) leads to the equations

$$
\begin{aligned}
\partial_{t} u-\Delta u=f(x) & \text { in } \Omega, \\
-\Delta u=f(x) & \text { in } B \backslash \bar{\Omega},
\end{aligned}
$$

Received March 15, 2007.

2000 Mathematics Subject Classification. Primary 35K65, 35B40, 35M10.

Key words and phrases. Parabolic-elliptic equation, degenerate parabolic equation, asymptotic behavior, sensitivity analysis.

E-mail address: bastian.gebauer@ricam.oeaw.ac.at

$W W W$ address: http://www.ricam.oeaw.ac.at/people/page/gebauer 

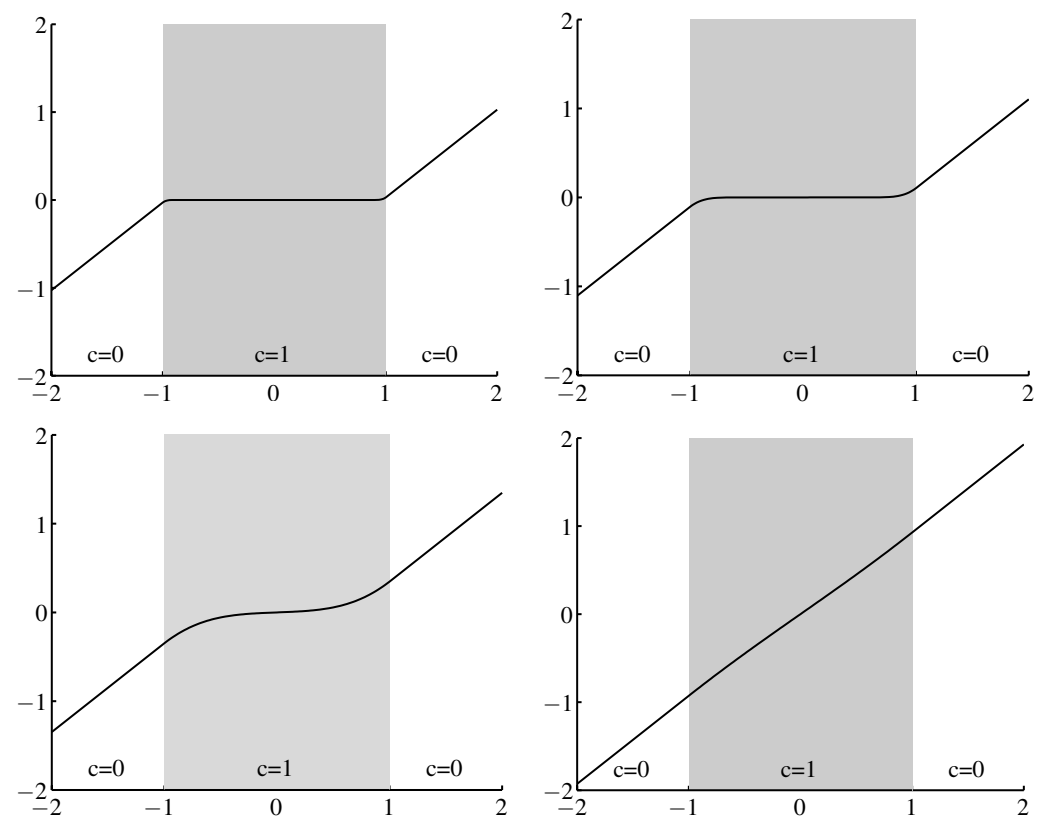

FIG. 1. Solution of a parabolic-elliptic equation

which are parabolic in $\Omega$ and elliptic in $B \backslash \bar{\Omega}$. (In fact for this choice of $c$ and $\kappa,(1.1)$ is equivalent to (1.2) and (1.3) together with some interface conditions on $\partial \Omega$; cf., e.g., $[6$, Lemma 2.2].) As an easy example, Figure 1 shows the solution of (1.1) with $B=]-2,2[$, $c=\chi_{]-1,1}, \kappa=1, f=0$, zero initial values and $\{-1,1\}$ as Neumann boundary values for $t \in\{0.001,0.01,0.1,1\}$.

Another important application of parabolic-elliptic problems appears in the illumination of conducting objects by low-frequency electromagnetic waves. Ammari, Buffa and Nédélec showed in [1] that the electric field $E$ generated by a current $J$ can be described in the low-frequency limit by

$$
\partial_{t}(\sigma(x) E(x, t))-\operatorname{curl}\left(\frac{1}{\mu(x)} \operatorname{curl} E(x, t)\right)=-\partial_{t} J(x, t)
$$

where $\mu(x)$ is the permeability and $\sigma(x)$ the electrical conductivity. The case of conducting objects in a non-conducting medium leads to a parabolic problem inside the conductors and an elliptic problems outside. The analogy to the heat equation is that the electric field is generated almost instantly in the non-conducting background, while it takes some time to build up inside the conductor due to eddy currents.

In situations with cylindrical symmetry (1.1) appears as the two-dimensional version of (1.4). For $B=\mathbb{R}^{2}$ and $\kappa=1$ this problem was studied by MacCamy and Suri in [10] and by Costabel, Ervin and Stephan in [3]. In both papers boundary integral operators are used to replace the Laplace equation in the exterior of $\Omega$ by a non-local boundary condition for the parabolic equation inside $\Omega$. This problem is then solved by a Galerkin method. In [2] Costabel uses boundary integral operators also to solve 
the resulting interior problem. In [6] Frühauf, Scherzer and the author have studied the inverse problem of detecting the interface between the parabolic and the elliptic part from boundary measurements.

All these works consider thermal capacities (resp. electrical conductivities) in the form of a characteristic function of a subset of $B$, so that the parabolic and the elliptic part are clearly separated. Also a rigorous justification for modeling a small thermal capacity (resp. electrical conductivity) by setting it to zero is still missing. The aim of this work is to close these two gaps by treating gradually vanishing capacities and analyzing the behavior of the solutions if the capacity tends to zero on a part of the domain.

In Section 2 we analyze equation (1.1) for general non-negative capacities $c(x) \in$ $L^{\infty}(B)$ and show in what sense one can speak of boundary and initial conditions. Then we prove the unique solvability of this problem in Section 3. The continuous dependence of the solutions from the capacity and a characterization of weak directional derivatives as solutions of related parabolic-elliptic problems is then studied in Section 4.

2. Analysis of the parabolic-elliptic problem. Let $B \subset \mathbb{R}^{n}$ be a smoothly bounded domain, $\left.T>0, B_{T}:=B \times\right] 0, T\left[, \kappa \in L_{+}^{\infty}(B), c \in L_{>}^{\infty}(B)\right.$, where we denote by $L_{+}^{\infty}$ the space of $L^{\infty}$-functions with positive (essential) infima and by $L_{\geq}^{\infty}$ the space of $L^{\infty}$-functions that are (almost everywhere) non-negative but not identical to zero.

We start by giving the equation (1.1) a rigorous mathematical meaning. Since $u$ and $\nabla u$ are multiplied with the non-smooth capacity $c$, resp. the conductivity $\kappa$, the equation does not make sense for general distributions $u$. In order to work in Hilbert space it seems natural to postulate that $u$ and $\nabla u$ are square-integrable functions. Thus we will look for a solution in the anisotropic Hilbert space $H^{1,0}(B)$, where $H^{r, s}(B)$ is defined for $r, s \geq 0$ by (see [8])

$$
H^{r, s}(B):=L^{2}\left(0, T, H^{r}(B)\right) \cap H^{s}\left(0, T, L^{2}(B)\right) .
$$

An analogous definition is used on $\partial B$ and for $s<\frac{1}{2}$ we will also use the dual spaces

$$
H^{-r,-s}(\partial B):=\left(H^{r, s}(\partial B)\right)^{\prime} .
$$

We further assume that $f \in L^{2}\left(B_{T}\right)$. If we take the derivatives in the sense of distributions, then for every $u \in H^{1,0}(B)$ our equation (1.1) makes sense and is equivalent to

$$
\begin{aligned}
& \int_{0}^{T} \int_{B}\left(c(x) u(x, t) \partial_{t} \varphi(x, t)-\kappa(x) \nabla u(x, t) \nabla \varphi(x, t)\right) \mathrm{d} x \mathrm{~d} t \\
& =\int_{0}^{T} \int_{B} f(x, t) u(x, t) \varphi(x, t) \mathrm{d} x \mathrm{~d} t
\end{aligned}
$$

for all $\varphi \in \mathcal{D}(B \times] 0, T[)$, where $\mathcal{D}(B \times] 0, T[)$ is the space of infinitely often differentiable functions with support in $B \times] 0, T$. We will also use the notation $\mathcal{D}(\bar{B} \times] 0, T[)$ for the restrictions of functions from $\mathcal{D}\left(\mathbb{R}^{n} \times\right] 0, T[)$ to $\left.B \times\right] 0, T[$ and the notation $\mathcal{D}(\bar{B} \times[0, T[)$ for the space of restrictions of functions from $\mathcal{D}\left(\mathbb{R}^{n} \times\right]-\infty, T[)$ to $\left.B \times\right] 0, T$. We will usually omit the arguments $x$ and $t$ and only use them where we expect them to improve readability. 
For solutions $u \in H^{1,0}(B)$ of (1.1) we can define Neumann traces $\left.\kappa \partial_{\nu} u\right|_{\partial B}$ by setting

$$
\left\langle\left.\kappa \partial_{\nu} u\right|_{\partial B}, \phi\right\rangle:=\int_{0}^{T} \int_{B}\left(\kappa \nabla u \cdot \nabla v_{\phi}-c u \partial_{t} v_{\phi}-f v_{\phi}\right) \mathrm{d} x \mathrm{~d} t
$$

for every function $\phi$ on $\partial B$ that has an extension $v_{\phi} \in \mathcal{D}(\bar{B} \times] 0, T[)$ with $\left.v_{\phi}\right|_{\partial B}=\phi$.

If $c$ vanishes in a neighborhood of $\partial B$, then $\kappa \partial_{\nu} u$ can obviously be extended to an element of $H^{-\frac{1}{2}, 0}(\partial B)$. In general, it has less temporal smoothness; cf., e.g., [2], where Costabel shows that for $c=1$ the Neumann trace operator is surjective onto $H^{-\frac{1}{2},-\frac{1}{4}}(\partial B)$. In order to obtain solvability also for vanishing $c$ we will only treat the case that

$$
\left.\kappa \partial_{\nu} u\right|_{\partial B}=g
$$

with $g \in H^{-\frac{1}{2}, 0}(\partial B)$.

To analyze in what sense solutions of (1.1) and (2.1) have well-defined initial values we will need to be very precise about the term "time-derivative". Recall that given a pair $(X, Y)$ of Banach spaces and a continuous injection $\iota: X \hookrightarrow Y$ a function $v \in L^{2}(0, T, X)$ has a time-derivative $\dot{v}=w \in L^{2}(0, T, Y)$ in the sense of vector-valued distributions if there exists $w \in L^{2}(0, T, Y)$ such that for all $\varphi \in \mathcal{D}(] 0, T[)$,

$$
\int_{0}^{T} w(t) \varphi(t) \mathrm{d} t=-\int_{0}^{T} \iota(v(t)) \dot{\varphi}(t) \mathrm{d} t
$$

(cf. e.g., [5, XVIII, §1, Def. 3]). In the case of a Gelfand triple

$$
V \hookrightarrow H \hookrightarrow V^{\prime}
$$

of separable real Hilbert spaces $H$ and $V$ the space

$$
W\left(0, T, V, V^{\prime}\right):=\left\{v \in L^{2}(0, T, V): \dot{v} \in L^{2}\left(0, T, V^{\prime}\right)\right\}
$$

is defined by taking the time derivative with respect to the injection $V \hookrightarrow V^{\prime}$, that is, the composition of $V \hookrightarrow H$ and $H \hookrightarrow V^{\prime}$. The choice of the injection $\iota$, resp. the choice of $H$, and the injections in the Gelfand triple is usually obvious and therefore omitted. However in our case a special choice for $H$ will be needed.

We first introduce the space

$$
W:=\left\{v \in H^{1,0}(B):(c v)^{\bullet} \in L^{2}\left(0, T, H^{1}(B)^{\prime}\right)\right\},
$$

where $(c v)^{\cdot}$ denotes the time-derivative of $c v \in L^{2}(B)$ in the sense of vector-valued distributions with respect to the (usual) injection

$$
L^{2}(B) \hookrightarrow H^{1}(B)^{\prime} .
$$

Now we define the space $L_{c}^{2}(B)$ by taking the closure of the set

$$
\{\sqrt{c} v: v \in \mathcal{D}(\bar{B})\} \subset L^{2}(B)
$$

with respect to the $L^{2}(B)$-norm. $L_{c}^{2}(B)$ is a Hilbert space equipped with the standard $L^{2}$ inner product. 
We can now show:

Lemma 2.1. If $u \in W$, then $\sqrt{c} u \in C\left(0, T, L_{c}^{2}(B)\right)$ and for all $u, v \in W$ the following integration by parts formula holds:

$$
\begin{aligned}
& \int_{0}^{T}\left\langle(c u)^{\cdot}(t), v(t)\right\rangle \mathrm{d} t+\int_{0}^{T}\left\langle(c)^{\cdot}(t), u(t)\right\rangle \mathrm{d} t \\
& =\int_{B} c(u(T) v(T)-u(0) v(0)) \mathrm{d} x .
\end{aligned}
$$

Proof. We will use the fact that for a Gelfand triple $V \hookrightarrow H \hookrightarrow V^{\prime}$ with injection $j: V \hookrightarrow H$ the image of the space $W\left(0, T, V, V^{\prime}\right)$ under $j$ is continuously imbedded in $C(0, T, H)$ and that for $u, v \in W\left(0, T, V, V^{\prime}\right)$ the integration by parts formula

$$
\begin{aligned}
& \int_{0}^{T}\langle\dot{u}(t), v(t)\rangle_{V^{\prime} \times V} \mathrm{~d} t+\int_{0}^{T}\langle\dot{v}(t), u(t)\rangle_{V^{\prime} \times V} \mathrm{~d} t \\
& =(j u(T), j v(T))_{H}-(j u(0), j v(0))_{H}
\end{aligned}
$$

holds; cf., e.g., [5, XVIII, §1, Theorems 1,2].

To this end we define the mapping

$$
j: H^{1}(B) \rightarrow L_{c}^{2}(B), \quad j v:=\sqrt{c} v .
$$

Obviously $j$ is continuous, has dense range and after identifying $L_{c}^{2}(B)$ with its dual, $j^{\prime}$ is given by

$$
j^{\prime}: L_{c}^{2}(B) \rightarrow\left(H^{1}(B)\right)^{\prime}, \quad\left\langle j^{\prime} w, v\right\rangle:=\int_{B} \sqrt{c} w v \mathrm{~d} x
$$

Unfortunately $j$ is not injective, so that $H^{1}(B) \rightarrow L_{c}^{2}(B) \rightarrow H^{1}(B)^{\prime}$ is not a Gelfand triple from which the assertion would follow immediately. However, after factoring out the nullspace of $j$ we obtain the Gelfand triple

$$
H^{1}(B) / N \hookrightarrow L_{c}^{2}(B) \hookrightarrow\left(H^{1}(B) / N\right)^{\prime},
$$

with $N:=\mathcal{N}(j)$ and the injections

$$
\begin{array}{rlrl}
\iota: H^{1}(B) / N & \rightarrow L_{c}^{2}(B), & \iota^{\prime}: L_{c}^{2}(B) & \rightarrow\left(H^{1}(B) / N\right), \\
\iota(v+N) & \left.:=\sqrt{c} v, \quad\left\langle\iota^{\prime} w, v+N\right)\right\rangle & :=\int_{B} \sqrt{c} w v \mathrm{~d} x .
\end{array}
$$

Now let $u \in W$ and $v=(c u)^{\circ} \in L^{2}\left(0, T, H^{1}(B)^{\prime}\right)$ be its time-derivative with respect to $L^{2}(B) \hookrightarrow H^{1}(B)^{\prime}$. For every $w \in N$ and $\varphi \in \mathcal{D}(] 0, T[)$ we have that

$$
\int_{0}^{T}\langle v(t), w\rangle_{H^{1}(B)^{\prime} \times H^{1}(B)} \varphi(t) \mathrm{d} t=-\int_{0}^{T} \int_{B} c u(t) w \mathrm{~d} x \dot{\varphi}(t) \mathrm{d} t=0,
$$


and thus $\langle v(t), w\rangle=0$ for $t \in] 0, T$ [ a. e. Hence $v(t) \in N^{\perp}$ and we can identify $v$ with an element of $L^{2}\left(0, T,\left(H^{1}(B) / N\right)^{\prime}\right)$, so that we obtain that for every $w+N \in H^{1}(B) / N$,

$$
\begin{aligned}
& \int_{0}^{T}\langle v(t), w+N\rangle_{\left(H^{1}(B) / N\right)^{\prime} \times H^{1}(B) / N} \varphi(t) \mathrm{d} t \\
& =-\int_{0}^{T} \int_{B} c u(t) w \mathrm{~d} x \dot{\varphi}(t) \mathrm{d} t \\
& =-\int_{0}^{T}\left\langle\iota^{\prime} \iota(u(t)+N), w+N\right\rangle_{\left(H^{1}(B) / N\right)^{\prime} \times H^{1}(B) / N} \varphi(t) \mathrm{d} t .
\end{aligned}
$$

This yields that $v=(u+N)^{\cdot}$ with respect to

$$
\iota^{\prime} \iota: H^{1}(B) / N \hookrightarrow\left(H^{1}(B) / N\right)^{\prime},
$$

so

$$
u+N \in W\left(0, T, H^{1}(B) / N,\left(H^{1}(B) / N\right)^{\prime}\right) .
$$

From the theorem stated in the beginning of this proof it now follows that

$$
\sqrt{c} u=\iota(u+N) \in C\left(0, T, L_{c}^{2}(B)\right)
$$

and that (2.4) holds.

Lemma 2.2. Every solution $u \in H^{1,0}(B)$ of (1.1) and (2.1) is in $W$ and thus has welldefined initial values

$$
\sqrt{c(x)} u(x, 0) \in L_{c}^{2}(B) \subseteq L^{2}(B) .
$$

For $t \in] 0, T\left[\right.$ a.e., $(c u)^{\circ}(t) \in H^{1}(B)^{\prime}$ is given by

$$
\left\langle(c u)^{\cdot}(t), w\right\rangle=\langle g(t), w\rangle+\int_{B}(f(x, t) w(x)-\kappa(x) \nabla u(x, t) \nabla w(x)) \mathrm{d} x
$$

for all $w \in H^{1}(B)$.

Proof. For $t \in] 0, T\left[\right.$ we define $v(t) \in H^{1}(B)^{\prime}$ by

$$
\langle v(t), w\rangle:=\langle g(t), w\rangle+\int_{B}(f(x, t) w(x)-\kappa(x) \nabla u(x, t) \nabla w(x)) \mathrm{d} x .
$$

It follows that $v \in L^{2}\left(0, T, H^{1}(B)^{\prime}\right)$, and from the definition of the Neumann derivative we conclude that for every $w \in H^{1}(B)$ and every $\varphi \in \mathcal{D}(] 0, T[)$,

$$
-\int_{0}^{T}\left(\int_{B} c(x) u(x, t) w(x) \mathrm{d} x\right) \partial_{t} \varphi(t) \mathrm{d} t=\int_{0}^{T}\langle v(t), w\rangle \varphi(t) \mathrm{d} t .
$$

This yields that $v=(c u)^{\cdot}$ with respect to $L^{2}(B) \hookrightarrow H^{1}(B)^{\prime}$ and thus $u \in W$.

Since the multiplication with $\sqrt{c}$ is a continuous operator from $L^{2}(B)$ to $L^{2}(B)$, the space $L_{c}^{2}(B)$ contains all functions of the form $\sqrt{c} v$ with $v \in L^{2}(B)$. Lemma 2.2 thus shows that the following problem makes sense:

For given $f \in L^{2}\left(B_{T}\right), g \in H^{-\frac{1}{2}, 0}(\partial B)$ and $u_{0} \in L^{2}(B)$ find $u \in H^{1,0}(B)$ such that

$$
\begin{aligned}
\partial_{t}(c u)-\nabla \cdot(\kappa \nabla u) & =f & & \text { in } B \times] 0, T[, \\
\kappa \partial_{\nu} u & =g & & \text { on } \partial B \times] 0, T[, \\
\sqrt{c} u(0) & =\sqrt{c} u_{0} & & \text { for } t=0 .
\end{aligned}
$$


Equation (2.7) can be interpreted as stating that wherever it makes sense to speak of initial values for $u$ they must agree with $u_{0}$. For the case $c=\chi_{\Omega}$ with some $\Omega \subset B$ this means that initial values are necessary, resp. meaningful, only on $\Omega$, i.e. where the equation is parabolic, but not for $B \backslash \bar{\Omega}$ where the equation is elliptic. This corresponds to the physical conception that in parts without heat capacity the domain instantly reaches its thermal equilibrium and thus has no memory.

We finish this section by giving an equivalent variational formulation to this problem.

Theorem 2.3. Let $f \in L^{2}\left(B_{T}\right), g \in H^{-\frac{1}{2}, 0}(\partial B)$ and $u_{0} \in L^{2}(B)$. The following four problems are equivalent:

(a) Find $u \in H^{1,0}(B)$ that solves (2.5), (2.6) and (2.7).

(b) Find $u \in W$ that solves (2.7) and

$$
\begin{aligned}
& \int_{0}^{T}\left\langle(c u)^{\cdot}, v\right\rangle \mathrm{d} t+\int_{0}^{T} \int_{B} \kappa \nabla u \cdot \nabla v \mathrm{~d} x \mathrm{~d} t \\
& =\int_{0}^{T}\left\langle g,\left.v\right|_{\partial B}\right\rangle \mathrm{d} t+\int_{0}^{T} \int_{B} f v \mathrm{~d} x \mathrm{~d} t
\end{aligned}
$$

for all $v \in H^{1,0}(B)$.

(c) Find $u \in H^{1,0}(B)$ that solves

$$
\begin{aligned}
& -\int_{0}^{T}\left\langle(c w)^{\cdot}, u\right\rangle \mathrm{d} t+\int_{0}^{T} \int_{B} \kappa \nabla u \cdot \nabla w \mathrm{~d} x \mathrm{~d} t \\
& =\int_{0}^{T}\left\langle g,\left.w\right|_{\partial B}\right\rangle \mathrm{d} t+\int_{0}^{T} \int_{B} f w \mathrm{~d} x \mathrm{~d} t+\int_{B} c(x) u_{0}(x) w(x, 0) \mathrm{d} x
\end{aligned}
$$

for all $w \in W$ with $\sqrt{c} w(\cdot, T)=0$.

(d) Find $u \in H^{1,0}(B)$ that solves

$$
\begin{aligned}
& -\int_{0}^{T} \int_{B} u c \partial_{t} \varphi \mathrm{d} x \mathrm{~d} t+\int_{0}^{T} \int_{B} \kappa \nabla u \cdot \nabla \varphi \mathrm{d} x \mathrm{~d} t \\
& =\int_{0}^{T}\left\langle g,\left.\varphi\right|_{\partial B}\right\rangle \mathrm{d} t+\int_{0}^{T} \int_{B} f \varphi \mathrm{d} x \mathrm{~d} t+\int_{B} c(x) u_{0}(x) \varphi(x, 0) \mathrm{d} x
\end{aligned}
$$

for all $\varphi \in \mathcal{D}(\bar{B} \times[0, T[)$.

Proof. We start by showing (a) $\Rightarrow$ (b). If $u \in H^{1,0}(B)$ solves (2.5) and (2.6), then Lemma 2.2 yields that $u \in W$ and that $(2.8)$ holds for all $v(x, t)=w(x) \varphi(t)$ with $w \in H^{1}(B)$ and $\varphi \in \mathcal{D}(] 0, T[)$. Since $H^{1}(B) \otimes \mathcal{D}(] 0, T[)$ is dense in $H^{1,0}(B)$ and both sides of (2.8) depend continuously on $v \in H^{1,0}(B)$, (b) follows.

(b) $\Rightarrow$ (c) follows from the integration by parts formula in Lemma 2.1.

We obtain (c) $\Rightarrow$ (d) from the fact that for $\varphi \in \mathcal{D}\left(\bar{B} \times\left[0, T[)\right.\right.$ the time-derivative $(c \varphi)^{\circ} \in$ $L^{2}\left(0, T, H^{1}(B)^{\prime}\right)$ of $c \varphi(t) \in L^{2}\left(B_{T}\right)$ with respect to the injection $L^{2}(B) \hookrightarrow H^{1}(B)^{\prime}$ is the image of the classical time-derivative $c(x) \partial_{t} \varphi(x, t)$ under this injection, i.e.

$$
\left.\left\langle(c \varphi)^{\cdot}(t), u(x, t)\right\rangle=\int_{B} c(x) \partial_{t} \varphi(x, t) u(x, t) \mathrm{d} x \quad \text { for } t \in\right] 0, T[\text { a.e. }
$$

In order to show $(\mathrm{d}) \Rightarrow(\mathrm{a})$ we first use $(\mathrm{d})$ with $\varphi \in \mathcal{D}(B \times] 0, T[)$ to obtain that $u$ solves (2.5) and then with $\varphi \in \mathcal{D}(\bar{B} \times] 0, T[)$ to obtain that $u$ solves (2.6). Lemma 2.2 then yields 
that $u \in W$. By using the integration by parts formula on $(2.10)$ with $\varphi(x, t)=\psi(x) \phi(t)$, where $\psi \in \mathcal{D}(\bar{B}), \phi \in \mathcal{D}([0, T[)$ and $\phi(0)=1$, it follows that

$$
\int_{B} \sqrt{c(x)} u_{0}(x) \sqrt{c(x)} \psi(x) \mathrm{d} x=\int_{B} \sqrt{c(x)} u(x, 0 \sqrt{c(x)} \psi(x) \mathrm{d} x .
$$

Since the set of all $\sqrt{c} \psi$ with $\psi \in \mathcal{D}(\bar{B})$ is dense in $L_{c}^{2}(B)$, this yields that $u$ solves $(2.7)$.

3. Existence and uniqueness. In this section we show that (2.5)-(2.7) has a unique solution. Though we can follow our approach from [6], several changes to the proofs are necessary to adapt them to this more general case:

Lemma 3.1. There is at most one $u \in H^{1,0}(B)$ that solves $(2.5)-(2.7)$.

Proof. Let $u \in H^{1,0}(B)$ be a solution of (2.5)-(2.7) with $f=0, g=0$ and $u_{0}=0$. By using Theorem 2.3(b) with $v:=u$ and the integration by parts formula from Lemma 2.2 we obtain

$$
\frac{1}{2} \int_{B} c u(T)^{2} \mathrm{~d} x+\int_{0}^{T} \int_{B} \kappa|\nabla u|^{2} \mathrm{~d} x \mathrm{~d} t=0 .
$$

This yields that $u$ is spatially constant in $B$, i.e. $u(x, t)=\psi(t)$ with a continuous real-valued function $\psi$. Since $c \not \equiv 0$ we obtain from $(2.5)$ that $\dot{\psi}(t)=0$ and from (2.7) that $\psi(0)=0$, so that $u(x, t)=\psi(t)=0$.

We will prove the existence using Lions's Projection Lemma:

Lemma 3.2 (Lions's Projection Lemma). Assume that $H$ is a Hilbert space and $\Phi$ is a subspace of $H$. Moreover let $a: H \times \Phi \rightarrow \mathbb{R}$ be a bilinear form satisfying the following properties:

a) For every $\varphi \in \Phi$, the linear form $u \mapsto a(u, \varphi)$ is continuous on $H$.

b) There exists $\alpha>0$ such that $a(\varphi, \varphi) \geq \alpha\|\varphi\|_{H}^{2}$ for all $\varphi \in \Phi$.

Then for each continuous linear form $l \in H^{\prime}$, there exists $u_{0} \in H$ such that

$$
a\left(u_{0}, \varphi\right)=\langle l, \varphi\rangle \quad \text { for all } \varphi \in \Phi \quad \text { and } \quad\left\|u_{0}\right\|_{H} \leq \frac{1}{\alpha}\|l\|_{H^{\prime}} .
$$

Proof. The lemma was proven in [7]. For the convenience of the reader we repeat our English translation from [6]:

From assumption a) and the Riesz Representation Theorem it follows that for every $\varphi \in \Phi$ there exists $K \varphi \in H$ with

$$
(u, K \varphi)=a(u, \varphi) \quad \text { for all } u \in H .
$$

This defines a linear (possibly unbounded) operator

$$
K: \Phi \rightarrow V:=K(\Phi) \subseteq H .
$$

From assumption b) it follows that $K$ is injective and thus possesses an inverse $R_{0}$ : $V \rightarrow \Phi$. Again using assumption b) we have

$$
\left\|R_{0} v\right\|^{2} \leq \frac{1}{\alpha} a\left(R_{0} v, R_{0} v\right)=\frac{1}{\alpha}\left(R_{0} v, v\right) \leq \frac{1}{\alpha}\left\|R_{0} v\right\|\|v\|,
$$


which yields $\left\|R_{0} v\right\| \leq \frac{1}{\alpha}\|v\|$. Thus $R_{0}$ can be extended by continuity to the closure $\bar{V}$ of $V$. If we denote this extension by $\bar{R}_{0}$, then we have $\bar{R}_{0}: \bar{V} \rightarrow \bar{\Phi}$.

$\bar{\Phi}$ is a closed subspace of the Hilbert space $H$ and thus also a Hilbert space. Using the Riesz Representation Theorem on $\bar{\Phi}$ we obtain a $\xi_{l} \in \bar{\Phi}$ with

$$
\langle l, \varphi\rangle=\left(\xi_{l}, \varphi\right) \quad \text { for all } \varphi \in \bar{\Phi} .
$$

Finally let $P: H \rightarrow \bar{V}$ be the orthogonal projection onto $\bar{V}$. Then $u_{0}:=P^{*} \bar{R}_{0}{ }^{*} \xi_{l}$ has the desired properties.

To prove existence of a solution and to study its continuity properties it is useful to introduce the spatially constant function

$$
u_{\mathbb{1}}(t):=\frac{1}{\int_{B} c \mathrm{~d} x}\left(\int_{0}^{t}\left(\left\langle g(s), \mathbb{1}_{\partial B}\right\rangle+\int_{B} f(x, s) \mathrm{d} x\right) \mathrm{d} s+\int_{B} c u_{0} \mathrm{~d} x\right),
$$

where $\mathbb{1}$ denotes the function that is identically one on the subset indicated in the subscript.

It is easily checked that $u$ solves $(2.5)-(2.7)$ if and only if $\tilde{u}:=u-u_{\mathbb{1}}$ solves

$$
\begin{aligned}
\partial_{t}(c \tilde{u})-\nabla \cdot(\kappa \nabla \tilde{u}) & =\tilde{f} & & \text { in } B \times] 0, T[, \\
\kappa \partial_{\nu} \tilde{u} & =\tilde{g} & & \text { on } \partial B \times] 0, T[, \\
\sqrt{c} \tilde{u}(0) & =\sqrt{c} \tilde{u}_{0} & & \text { for } t=0,
\end{aligned}
$$

with

$$
\begin{aligned}
\tilde{f} & =f-\frac{c}{\int_{B} c \mathrm{~d} x}\left(\left\langle g(t), \mathbb{1}_{\partial B}\right\rangle-\int_{B} f(x, t) \mathrm{d} x\right), \\
\tilde{g} & =g, \\
\tilde{u}_{0} & =u_{0}-\frac{1}{\int_{B} c \mathrm{~d} x} \int_{B} c u_{0} \mathrm{~d} x .
\end{aligned}
$$

Physically this corresponds to normalizing $u$ to a function $\tilde{u}$, whose average temperature $\int_{B} c \tilde{u} \mathrm{~d} x$ stays zero. Now we can show:

Theorem 3.3. Let $f \in L^{2}\left(B_{T}\right), g \in H^{-\frac{1}{2}, 0}(\partial B), u_{0} \in L^{2}(B)$ and $\tilde{f}, \tilde{g}$ and $\tilde{u}_{0}$ be defined by (3.4)-(3.6). There exists a solution $\tilde{u} \in H^{1,0}(B)$ of (3.1)-(3.3) and thus a solution $u=\tilde{u}+u_{\mathbb{1}} \in H^{1,0}(B)$ of $(2.5)-(2.7)$.

The solution $u \in H^{1,0}(B)$ depends continuously on $f, g$ and $u_{0}$ (with a continuity constant that depends on $c$ ).

Proof. We define the spaces

$$
\begin{aligned}
H_{\square}^{1}(B) & :=\left\{v \in H^{1}(B): \int_{B} c v \mathrm{~d} x=0\right\}, \\
H & :=\left\{v \in L^{2}\left(0, T, H_{\square}^{1}(B)\right): \sqrt{c} v(0) \in L^{2}(B)\right\}, \\
\Phi & :=\left\{\varphi \in \mathcal { D } \left(\left[0, T[\times \bar{B}): \int_{B} c \varphi \mathrm{d} x=0\right\},\right.\right.
\end{aligned}
$$


and set for all $v \in H$ and $\varphi \in \mathcal{D}([0, T[\times \bar{B})$,

$$
\begin{aligned}
a(v, \varphi) & :=\int_{0}^{T} \int_{B} \kappa \nabla v \cdot \nabla \varphi \mathrm{d} x \mathrm{~d} t-\int_{0}^{T} \int_{B} v c \partial_{t} \varphi \mathrm{d} x \mathrm{~d} t \\
\langle l, v\rangle & :=\int_{0}^{T}\left\langle\tilde{g},\left.v\right|_{\partial B}\right\rangle \mathrm{d} t+\int_{0}^{T} \int_{B} \tilde{f} v \mathrm{~d} x \mathrm{~d} t+\int_{B} c(x) \tilde{u}_{0}(x) v(x, 0) \mathrm{d} x,
\end{aligned}
$$

where $\tilde{f}, \tilde{g}$ and $\tilde{u}_{0}$ are defined by (3.4)-(3.6). Note that we defined $H$ in a common but sloppy way, since $\sqrt{c} v(0)$ is not well defined for functions in $L^{2}\left(0, T, H_{\square}^{1}(B)\right)$. A rigorous definition is obtained by taking the closure of $\mathcal{D}([0, T] \times \bar{B})$ with respect to

$$
\|\varphi\|_{H}^{2}:=\|\varphi\|_{L^{2}\left(0, T, H^{1}(B)\right)}^{2}+\|\sqrt{c} \varphi(0)\|_{L^{2}(B)}^{2} .
$$

This yields a Hilbert space which is continuously imbedded in $H^{1,0}(B)$, so that $\varphi(x, t) \mapsto$ $\int_{B} c(x) \varphi(x, t) \mathrm{d} x \in L^{2}(] 0, T[)$ defines a continuous mapping. $H$ is then defined as the kernel of this mapping.

With the integration by parts formula in Lemma 2.2 it follows that

$$
a(\varphi, \varphi)=\int_{0}^{T} \int_{B} \kappa|\nabla \varphi|^{2} \mathrm{~d} x \mathrm{~d} t+\int_{B} c(x) \varphi(x, 0)^{2} \mathrm{~d} x .
$$

From $\kappa \in L_{+}^{\infty}(B)$ and Poincaré's inequality (cf., e.g., Lemma 4.1 in Section 4) we obtain a constant $\alpha>0$ such that

$$
a(\varphi, \varphi) \geq \alpha\|\varphi\|_{H}^{2} \quad \text { for all } \varphi \in \Phi .
$$

Obviously $l \in H^{\prime}$ and $\|l\|_{H^{\prime}}$ depends continuously on $\tilde{f}, \tilde{g}$ and $\tilde{u}_{0}$, which in turn depend continuously on $f, g$ and $u_{0}$. Lions's Projection Lemma (Lemma 3.2) thus yields the existence of a $\tilde{u}$ that solves

$$
a(\tilde{u}, \varphi)=l(\varphi) \quad \text { for all } \varphi \in \Phi,
$$

and $\tilde{u}$ depends continuously on $f, g$ and $u_{0}$.

From $\int_{B} c(x) \tilde{u}(x, t) \mathrm{d} x=0$ for $\left.t \in\right] 0, T\left[\right.$ a. e. and the definition of $\tilde{f}, \tilde{g}$ and $\tilde{u}_{0}$ it follows that

$$
a(\tilde{u}, \varphi)=0=l(\varphi)
$$

for all spatially constant $\varphi \in \mathcal{D}([0, T[\times \bar{B})$. Since $\mathcal{D}([0, T[\times \bar{B})$ is the sum of $\Phi$ and the space of spatially constant functions, it follows that $\tilde{u}$ fulfills $(3.7)$ for all $\varphi \in \mathcal{D}([0, T[\times \bar{B})$. Theorem 2.3(d) now yields that $\tilde{u}$ solves $(3.1)-(3.3)$, so $u:=\tilde{u}+u_{\mathbb{1}}$ solves $(2.5)-(2.7)$ and since $u_{\mathbb{1}}$ depends continuously on $f, g$ and $u_{0}$, also $u$ does.

\section{Sensitivity analysis.}

4.1. Results for general data. We will now study the dependence of the solution $u$ from the capacity $c$. For ease of presentation we write $\left(c_{n}\right)$ instead of $\left(c_{n}\right)_{n \in \mathbb{N}}$ for a sequence indexed over natural numbers and we keep $\kappa \in L_{+}^{\infty}(B), f \in L^{2}\left(B_{T}\right), g \in H^{-\frac{1}{2}, 0}(\partial B)$ and $u_{0} \in L^{2}(B)$ fixed. The results in this section all easily extend to the cases where these parameters are also variable.

Using the variational formulation in Theorem 2.3(b) one obtains a bound for the gradient $\nabla u$ of the solution $u$ and after decomposing $u=\tilde{u}+u_{\mathbb{1}}$ as in Section 3 we can 
work with a normalized $\tilde{u}$ (in the sense that $\int_{B} c \tilde{u} \mathrm{~d} x=0$ ), so that $\nabla \tilde{u}$ and $\tilde{u}$ are related by a Poincaré inequality. However, this normalization depends on $c$. Therefore we will need a formulation of Poincaré's inequality that explicitly contains the normalization. The following formulation was used by Lukaschewitsch, Maass and Pidcock in [9] in the context of weighted Sobolev spaces:

Lemma 4.1. Let $l \in H^{1}(B)^{\prime}$ be a normalizing functional, i.e. $l\left(\mathbb{1}_{B}\right) \neq 0$. Then there exists a constant $C>0$ such that

$$
\|v\|_{H^{1}(B)}^{2} \leq C\left(\|\nabla v\|_{L^{2}(B)}^{2}+l(v)^{2}\right) \quad \text { for all } v \in H^{1}(B) .
$$

Proof. From the standard form of the Poincaré inequality (cf., e.g., [4, IV, §7, Prop. 2]) one obtains that the lemma holds for $\tilde{l}(v):=\int_{B} v \mathrm{~d} x$, and so there exists $C^{\prime}>0$ such that

$$
\|v\| \leq C^{\prime}\left(\|\nabla v\|_{L^{2}(B)}+\tilde{l}(v)\right) \quad \text { for all } v \in H^{1}(B)
$$

Since every $v \in H^{1}(B)$ can be written as $v=a \mathbb{1}_{B}+w$ with $a \in \mathbb{R}, w \in H^{1}(B)$ and $\tilde{l}(w)=0$ we conclude that with $C^{\prime \prime}:=\frac{\tilde{l}(\mathbb{1})}{l(\mathbb{1})}$,

$$
\begin{aligned}
\tilde{l}(v) & =a \tilde{l}(\mathbb{1})=C^{\prime \prime} l(a \mathbb{1}) \leq C^{\prime \prime}(l(v)+|l(w)|) \\
& \leq C^{\prime \prime}\left(l(v)+\|l\|_{H^{1}(B)^{\prime}}\|w\|_{H^{1}(B)}\right) \\
& \leq C^{\prime \prime}\left(l(v)+C^{\prime}\|l\|_{H^{1}(B)^{\prime}}\|\nabla w\|_{L^{2}(B)}\right) \\
& =C^{\prime \prime}\left(l(v)+C^{\prime}\|l\|_{H^{1}(B)^{\prime}}\|\nabla v\|_{L^{2}(B)}\right)
\end{aligned}
$$

from which the assertion follows.

Now we follow the procedure outlined in the beginning of this section to obtain an upper bound on the solutions:

Lemma 4.2. Let $c \in L_{\ngtr}^{\infty}(B)$. There exists $C>0$ such that for every sequence

$$
\left(c_{n}\right) \subset L_{\gtrless}^{\infty}(B) \quad \text { with } \quad \lim _{n \rightarrow \infty} c_{n}=c
$$

the corresponding solutions $\left(u_{n}\right)$ and $u$ satisfy

$$
\limsup _{n \rightarrow \infty}\left\|u_{n}\right\|_{H^{1,0}(B)} \leq C \quad \text { and } \quad \limsup _{n \rightarrow \infty}\left\|\left(c_{n} u_{n}\right)^{\cdot}\right\|_{L^{2}\left(0, T, H^{1}(B)^{\prime}\right)} \leq C .
$$

Proof. We decompose $u=\tilde{u}+u_{\mathbb{1}}$ and $u_{n}=\tilde{u}_{n}+u_{\mathbb{1}}^{(n)}$ as in Section 3. From the definition of $u_{\mathbb{1}}$ and $u_{\mathbb{1}}^{(n)}$ it is obvious that $u_{\mathbb{1}}^{(n)} \rightarrow u_{\mathbb{1}}$ in $H^{1,0}(B)$.

Now we use the variational formulation in Theorem 2.3(b) and obtain a $C^{\prime}>0$ such that

$$
\left\|\nabla \tilde{u}_{n}\right\|_{L^{2}\left(B_{T}\right)}=\left\|\nabla u_{n}\right\|_{L^{2}\left(B_{T}\right)} \leq C^{\prime}\left(1+\left\|c_{n}\right\|_{L^{\infty}(B)}\right) \quad \text { for all } n \in \mathbb{N} .
$$


Using Poincaré's inequality in Lemma 4.1 there exist $C^{\prime \prime}, C^{\prime \prime \prime}>0$ such that for all $n \in \mathbb{N}$,

$$
\begin{aligned}
\left\|\tilde{u}_{n}\right\|_{H^{1,0}(B)}^{2} & \leq C^{\prime \prime}\left(\left\|\nabla \tilde{u}_{n}\right\|_{L^{2}\left(B_{T}\right)}^{2}+\int_{0}^{T}\left(\int_{B} c \tilde{u}_{n}\right)^{2} \mathrm{~d} x \mathrm{~d} t\right) \\
& =C^{\prime \prime}\left(\left\|\nabla \tilde{u}_{n}\right\|_{L^{2}\left(B_{T}\right)}^{2}+\int_{0}^{T}\left(\int_{B}\left(c-c_{n}\right) \tilde{u}_{n}\right)^{2} \mathrm{~d} x \mathrm{~d} t\right) \\
& \leq C^{\prime \prime \prime}\left(\left\|\nabla \tilde{u}_{n}\right\|_{L^{2}\left(B_{T}\right)}^{2}+\left\|c-c_{n}\right\|_{L^{\infty}(B)}^{2}\left\|\tilde{u}_{n}\right\|_{L^{2}\left(B_{T}\right)}^{2}\right)
\end{aligned}
$$

and so the first assertion follows.

From the explicit form for $(\mathrm{cu})^{\cdot}$ in Lemma 2.2 it follows that $(\mathrm{cu})^{\cdot}$ depends continuously on $u$ with a constant independent of $c$, so that the second assertion also holds.

THEOREM 4.3. Let

$$
\left(c_{n}\right) \subset L_{\gtrless}^{\infty}(B), \quad c \in L_{\gtrless}^{\infty}(B) \quad \text { with } \quad \lim _{n \rightarrow \infty} c_{n}=c
$$

and $\left(u_{n}\right), u$ be the corresponding solutions.

Then $\left(u_{n}\right)$ converges weakly against $u$ in $H^{1,0}(B)$ and $\left(c_{n} u_{n}\right)^{*}$ converges weakly against $(c u)^{\cdot}$ in $L^{2}\left(0, T, H^{1}(B)^{\prime}\right)$.

Proof. To prove the weak convergence of $\left(u_{n}\right)$ it suffices to show that every subsequence of $\left(u_{n}\right)$ has a subsequence that converges weakly against $u$. From Lemma 4.2 we know that $\left(u_{n}\right)$ is bounded, so that every subsequence of $\left(u_{n}\right)$ contains a subsequence (that we still denote by $\left(u_{n}\right)$ for ease of notation) such that $u_{n} \rightarrow v$ in $H^{1,0}(B)$ with some $v \in H^{1,0}(B)$. From Theorem 2.3(d) it follows immediately that $v$ solves the parabolicelliptic equations (2.5), (2.6) and (2.7) with capacity $c$, so that by uniqueness $v=u$.

As in the proof of Lemma 4.2 the weak convergence of $\left(c_{n} u_{n}\right)^{\circ}$ against $(c u)^{\circ}$ follows from that of $\left(u_{n}\right)$ against $u$ by the explicit form of $\left(c_{n} u_{n}\right)^{\circ}$ and $(c u)^{\circ}$ in Lemma 2.2 .

4.2. Results for additional temporal smoothness. We obtain a stronger result under additional assumptions on the temporal regularity of the solution. For this we assume in this subsection that we are given

$$
f \in H^{1}\left(0, T, L^{2}(B)\right), \quad g \in H^{1}\left(0, T, H^{-\frac{1}{2}}(\partial B)\right) \quad \text { and } \quad u_{0} \in H^{1}(B)
$$

with $\kappa \partial_{\nu} u_{0}=g(0)$ and $\nabla \cdot \kappa \nabla u_{0}+f(0)=0$. (Note that for the next lemma the last condition can be replaced by the weaker but $c$-dependent assumption that there exists $\tilde{u}_{0} \in L^{2}(B)$ with $\sqrt{c} \tilde{u}_{0}=\sqrt{c} u_{0}$ and $\nabla \cdot \kappa \nabla u_{0}+f(0) \in L_{c}^{2}(B)$.)

LEMma 4.4. For every solution $u \in H^{1,0}(B)$ of (2.5), (2.6) and (2.7) it follows that $u \in H^{1}\left(0, T, H^{1}(B)\right), u(0)=u_{0}$, and $\dot{u} \in H^{1,0}(B)$ solves

$$
\begin{aligned}
\partial_{t}(c \dot{u})-\nabla \cdot(\kappa \nabla \dot{u}) & =\dot{f} & & \text { in } B \times] 0, T[, \\
\kappa \partial_{\nu} \dot{u} & =\dot{g} & & \text { on } \partial B \times] 0, T[, \\
\sqrt{c} \dot{u}(0) & =0 & & \text { for } t=0 .
\end{aligned}
$$


Proof. The proof is analogous to showing time regularity for parabolic equations (cf., e.g., [11, Sect. 10.1.4]). From Section 3 we know that (4.1)-(4.3) have a unique solution $v \in H^{1,0}(B)$, so it only remains to show that $\dot{u}=v$ or equivalently that

$$
z(t):=u_{0}+\int_{0}^{t} v(s) \mathrm{d} s
$$

solves the equation in Theorem $2.3(\mathrm{~d})$. This can be easily shown by first verifying the equation for $\varphi=\dot{\psi}$ with $\psi \in \mathcal{D}(\bar{B} \times[0, T[)$ and then for functions $\varphi(x) \in \mathcal{D}(\bar{B})$ that are constant in time.

LEMMA 4.5. Let $\left(c_{n}\right) \subset L_{\gtrless}^{\infty}(B), c \in L_{\gtrless}^{\infty}(B)$ and $\left(u_{n}\right), u$ be the corresponding solutions. Then $u-u_{n} \in H^{1,0}(B)$ solves

$$
\begin{aligned}
\partial_{t}\left(c\left(u-u_{n}\right)\right)-\nabla \cdot\left(\kappa \nabla\left(u-u_{n}\right)\right) & =\left(c_{n}-c\right) \dot{u}_{n} & & \text { in } B \times] 0, T[, \\
\kappa \partial_{\nu}\left(u-u_{n}\right) & =0, & & \text { on } \partial B \times] 0, T[, \\
\sqrt{c}\left(u-u_{n}\right)(0) & =0 & & \text { for } t=0 .
\end{aligned}
$$

Proof. It is easily seen that $u_{n} \in H^{1}\left(0, T, L^{2}(B)\right)$ yields that

$$
\left(\left(c_{n}-c\right) u_{n}\right)^{\cdot}=\left(c_{n}-c\right) \dot{u}_{n} \in L^{2}\left(0, T, L^{2}(B)\right) \hookrightarrow L^{2}\left(0, T, H^{1}(B)^{\prime}\right)
$$

with respect to the injection $L^{2}(B) \hookrightarrow H^{1}(B)^{\prime}$.

With this we obtain from Theorem $2.3(\mathrm{~d})$ that $u-u_{n}$ solves

$$
\begin{aligned}
& -\int_{0}^{T} \int_{B}\left(u-u_{n}\right) c \partial_{t} \varphi \mathrm{d} x \mathrm{~d} t+\int_{0}^{T} \int_{B} \kappa \nabla\left(u-u_{n}\right) \cdot \nabla \varphi \mathrm{d} x \mathrm{~d} t \\
& =-\int_{0}^{T} \int_{B} u_{n}\left(c_{n}-c\right) \partial_{t} \varphi \mathrm{d} x \mathrm{~d} t+\int_{B}\left(c-c_{n}\right)(x) u_{0}(x) \varphi(x, 0) \mathrm{d} x \\
& =\int_{0}^{T} \int_{B} \dot{u}_{n}\left(c_{n}-c\right) \varphi \mathrm{d} x \mathrm{~d} t+\int_{B} c\left(u_{0}(x)-u_{n}(x, 0)\right) \varphi(x, 0) \mathrm{d} x
\end{aligned}
$$

for all $\varphi \in \mathcal{D}\left(\bar{B} \times\left[0, T[)\right.\right.$. From Lemma 4.4 we know that $u_{n}(x, 0)=u_{0}(x)$, so the assertion follows.

We can now show that the solutions depend continuously (in the strong sense) on the capacity and characterize the weak one-sided directional derivatives of the solution with respect to capacity.

THEOREM 4.6. Let $c \in L_{\gtrless}^{\infty}(B)$.

(a) There exists $C>0$ such that for every sequence

$$
\left(c_{n}\right) \subset L_{\gtrless}^{\infty}(B) \quad \text { with } \quad \lim _{n \rightarrow \infty} c_{n}=c
$$

the corresponding solutions $\left(u_{n}\right)$ and $u$ satisfy

$$
\limsup _{n \rightarrow \infty} \frac{\left\|u_{n}-u\right\|_{H^{1,0}(B)}}{\left\|c_{n}-c\right\|_{L^{\infty}(B)}} \leq C .
$$

In particular $\left(u_{n}\right)$ converges (strongly) against $u$ in $H^{1,0}(B)$. 
(b) Let $d \in L^{\infty}(B),\|d\|=1$ and $c+h d \geq 0$ for sufficiently small $h>0$ and denote by $u_{c}, u_{c+h d}$ the solutions to capacity $c$, resp. $c+h d$. Then for $h \rightarrow 0^{+}$,

$$
\frac{1}{h}\left(u_{c+h d}-u_{c}\right)
$$

converges weakly against the solution $v \in H^{1,0}(B)$ of

$$
\partial_{t}(c v)-\nabla \cdot(\kappa \nabla v)=-d \dot{u}
$$

with zero initial and Neumann boundary data.

Proof. From Lemma 4.4 and Lemma 4.2 it follows that $\left(\dot{u}_{n}\right)$ is bounded in $H^{1,0}(B)$, so Lemma 4.5 together with the continuous dependence of the solution on the data (see Theorem 3.3) yields assertion (a).

Likewise it follows from Lemma 4.4 and Theorem 4.3 that $\left(\dot{u}_{n}\right)$ converges weakly against $\dot{u}$ in $H^{1,0}(B)$, so Lemma 4.5 and the continuous linear dependence of the solution on the data also yield assertion (b).

\section{REFERENCES}

[1] H. Ammari, A. Buffa, J.-C. Nédélec, A justification of eddy currents model for the Maxwell equations, SIAM J. Appl. Math. 60 (2000), 1805-1823. MR1761772 (2001g:78003)

[2] M. Costabel, Boundary integral operators for the heat equation, Integral Equations Oper. Theory 13 (1990), 488-552. MR1058085 (91j:35119)

[3] M. Costabel, V. J. Ervin, E. P. Stephan, Symmetric coupling of finite elements and boundary elements for a parabolic-elliptic interface problem, Quart. Appl. Math. 48 (1990), 265-279. MR1052136 (92e:65145)

[4] R. Dautray, J. L. Lions, Mathematical analysis and numerical methods for science and technology - volume 2: Functional and variational methods, Springer-Verlag, Berlin, 2000. MR969367 (89m:00001)

[5] R. Dautray, J.-L. Lions, Mathematical analysis and numerical methods for science and technology volume 5: Evolution problems I, Springer-Verlag, Berlin, Heidelberg, 2000. MR1156075 (92k:00006)

[6] F. Frühauf, B. Gebauer, O. Scherzer, Detecting interfaces in a parabolic-elliptic problem from surface measurements, SIAM J. Numer. Anal. 45 (2007), 810-836.

[7] J. L. Lions, Equations différentielles opérationnelles et problèmes aux limites, Springer-Verlag, Berlin, 1961. MR0153974 (27:3935)

[8] J. L. Lions, E. Magenes, Non-homogeneous boundary value problems and applications II, Grundlehren Math. Wiss., vol. 182, Springer-Verlag, Berlin, Heidelberg, New York, 1972.

[9] M. Lukaschewitsch, P. Maass, M. Pidcock, Tikhonov regularization for electrical impedance tomography on unbounded domains, Inverse Probl. 19 (2003), 585-610. MR1984879 (2004f:65079)

[10] R. C. MacCamy, M. Suri, A time-dependent interface problem for two-dimensional eddy currents, Quart. Appl. Math. 44 (1987), 675-690. MR872820 (87m:78007)

[11] M. Renardy, R. C. Rogers, An introduction to partial differential equations, Texts Appl. Math., vol. 13, Springer-Verlag, New York, 1993. MR1211418 (94c:35001) 